\title{
EMMNeurolesy open Experiences of remote consulting for patients and neurologists during the COVID-19 pandemic in Scotland
}

\author{
Maria Stavrou, ${ }^{1,2,3}$ Eva Lioutas, ${ }^{4}$ Joanna Lioutas, ${ }^{4}$ Richard J Davenport ${ }^{1,2,3}$
}

To cite: Stavrou M, Lioutas E, Lioutas J, et al. Experiences of remote consulting for patients and neurologists during the COVID-19 pandemic in Scotland. BMJ Neurology Open 2021;3:e000173. doi:10.1136/ bmjno-2021-000173

- Additional supplemental material is published online only. To view, please visit the journal online (http://dx.doi.org/10. 1136/bmjno-2021-000173).

Received 03 May 2021 Accepted 26 0ctober 2021

Check for updates

(C) Author(s) (or their employer(s)) 2021. Re-use permitted under CC BY-NC. No commercial re-use. See rights and permissions. Published by BMJ.

${ }^{1}$ Centre for Clinical Brain

Sciences, University of

Edinburgh, Edinburgh, UK

${ }^{2}$ Department of Clinical

Neurosciences, NHS Lothian,

Edinburgh, UK

${ }^{3}$ Anne Rowling Regenerative

Neurology Clinic, Edinburgh, UK

${ }^{4}$ University of Edinburgh,

Edinburgh, UK

Correspondence to

Dr Maria Stavrou;

mstavrou@ed.ac.uk

\section{ABSTRACT}

Background Remote consulting is an emerging model in managing chronic neurological conditions and has been widely implemented during the COVID-19 pandemic. The objective of this national survey was to investigate the initial experiences of remote consulting for neurologists and patients with established neurological conditions under follow-up during the first COVID-19 phase. Methods In collaboration with the Scottish Association of Neurological Sciences and the Neurological Alliance of Scotland, we conducted a web-based survey of neurologists and patients between October and November 2020.

Findings Data was available for 62 neurologists and 201 patients. The consensus among neurologists was that remote consulting is a satisfactory way of delivering healthcare in selected groups of patients. For practical and technical reasons, there was preference for phone over video consultations (phone $63 \%$ vs video $33 \%, p=0.003$ ). The prevailing opinion among clinicians was that considerable training interventions for remote consultation skills are required ('yes' $63 \%$ vs 'no' $37 \%, p=0.009$ ) to improve clinician consultation skills and successfully embed this new model of care.

Most patients perceived remote consultations as safe, effective and convenient, with $89 \%$ of patients being satisfied with their remote consultation experience. Although traditional face-to-face consultations were the favoured way of interaction for $62 \%$ of patients, a significant proportion preferred that some of their future consultations be remote.

Interpretation Although not a replacement for faceto-face consultations, this survey illustrates that remote consulting can be an acceptable adjunct to traditional face-to-face consultations for doctors and patients. More research is required to identify overall safety and applicability.

\section{INTRODUCTION}

Telemedicine (remote consulting) is defined as the exchange of medical information and delivery of medical care through electronic, digital and internet-based or telephone-based communication. Pre-pandemic, the main benefit of remote consulting was providing subspecialty healthcare to rural or underserved populations, where reducing patient travel for hospital visits was seen as a way to improve equity of service delivery ${ }^{1}$ and achieve better access for patients with impaired mobility or other disabilities (eg, cognitive) from chronic neurological conditions. ${ }^{2-4}$

Remote consulting has been a wellestablished care model in neurology for over 10 years, ${ }^{5}$ primarily for stroke treatment. Stroke telemedicine has enabled accessibility to acute stroke care and timely administration of thrombolysis with improved patient outcomes such as independent ambulation and reduction in disability days. ${ }^{6}$ More recently, it has evolved as a model in chronic neurological conditions including Parkinson's disease, multiple sclerosis and epilepsy. ${ }^{7-11} \mathrm{~A}$ study by Qiang and Marras ${ }^{12}$ implementing remote consulting for patients with Parkinson's disease demonstrated high patient satisfaction due to improved convenience, accessibility and reduced caregiver burden. Additionally, for patients with multiple sclerosis, remote consulting enhanced medication compliance. ${ }^{11}$

Despite the benefits of remote consulting, there are drawbacks. It is a less personable medium, which challenges the building of rapport in the clinician-patient relationship. ${ }^{9}{ }^{13}$ As it can be difficult or impossible to perform clinical examination via remote consulting, it lends itself best to history-driven conditions. The implications of missing key information or clinical signs from remote consulting remain to be examined in the context of patient safety and quality of care. Additionally, remote consulting comes with risks associated with the digital divide among patients ${ }^{14}$; alongside technical, logistical and regulatory challenges. ${ }^{9}$

The COVID-19 pandemic has forced a huge shift towards remote consultations. ${ }^{15}$ Physicians and healthcare systems worldwide have adopted remote treatment approaches that obviate the need for physical contact between patients and healthcare providers, thus reducing risk of viral transmission. Many 
of these changes and models of care will likely persist after the pandemic-sustainable adaptations and enhancement of remote delivery systems are therefore crucial to optimise healthcare provision and mitigate inconsistencies in care.

The goal of this survey was to record the experiences and opinions of remote consulting among both neurologists and patients with neurological conditions across Scotland. We sought to explore the opportunities and challenges for integrating remote consulting in the management of neurological conditions.

\section{METHODS \\ Design and setting}

In March 2020, the Scottish Government instructed specialists working in hospitals in Scotland to conduct all non-emergency outpatient consultations remotely, in an effort to prevent COVID-19 transmission. This decision was supported by the Association of British Neurologists and other specialty associations through the Royal College of Physicians. Face-to-face consultations only occurred for urgent neurology cases; however, there was massive variation across Scotland as uniform guidelines regarding remote versus in-person consultations did not exist this early on in the pandemic. Close liaison with primary care was necessary to prevent admissions and maintain support for patients with long-term neurological conditions.

We conducted two separate surveys to investigate neurology remote consulting during the COVID-19 pandemic, from the perspectives of both neurologists and patients.

In collaboration with the Scottish Association of Neurological Sciences and the Neurological Alliance of Scotland, the surveys were: (1) distributed to neurologists via email, and (2) to neurology patients with established neurological conditions under regular follow-up via publication on social media, across Scotland. The Neurological Alliance of Scotland is an umbrella body of nonprofit organisations representing and supporting people with neurological conditions in Scotland ${ }^{16}$; each group was invited to distribute and promote the online survey to its members.

The patient survey was completed anonymously, while the clinician survey was completed with discretionary name disclosure. Both surveys were implemented online during the COVID-19 pandemic, between October and November 2020. During this time period, the out-ofhospital measures described above remained in effect. At the time of writing, Scotland remains in a full lockdown with a 'stay at home' order that has been in effect since 5 January 2021, ${ }^{17}$ although these restrictions are slowly being eased.

\section{Questionnaires and outcomes}

The clinician survey assessed experiences and opinions on remote consulting during the COVID-19 pandemic-including the effect on job satisfaction, confidence in examination findings and perceived advantages and challenges compared with face-to-face review. The clinicians were also asked for views on the future of remote consulting and implications on training.

The patient survey assessed experiences and opinions on remote consulting during the COVID-19 pandemicincluding which methods they had experienced, which they preferred and rating overall satisfaction with remote consulting. We collected background variables including age, sex, and neurological condition.

\section{Statistical analyses}

For descriptive data, proportions and means are provided. Categorical data from the clinician dataset were compared using the $\chi^{2}$ test. For the patient dataset, we undertook multivariable logistic regression with the following dependent variables:

1. Remote consultation preference: phone versus video.

2. Concerns about the effectiveness of remote consultations.

3. Lack of familiarity with the technology.

4. Preference for some future consultations to be undertaken remotely rather than face-to-face.

5. Satisfaction with remote consultations.

Age, sex, neurological conditions, and language were included as independent variables.

The following conditions with adequately reported data (minimum of 10 patients per condition) were selected for the multivariable logistic regression models: dystonia, epilepsy, functional neurological disorder (FND), multiple sclerosis, myasthenia gravis and Parkinson's disease.

The reference group in dummy coding was chosen for statistical reasons to minimise the variance of estimates. Since all reference groups were sufficiently common (eg, prevalence of 13\%-91\%), selecting a different group would make no difference to the statistical inference.

Statistical significance was defined by $\mathrm{p}<0.05$ using a two-sided test.

Statistical analyses were undertaken using the statsmodel package in Python 3.8.

\section{RESULTS}

\section{Patients}

In total, 201 patients ( $67 \%$ female; $32 \%$ male) participated in the national survey. The characteristics of the patient respondents are shown in figure 1, their neurological conditions in figure 2. The most common neurological conditions were myasthenia gravis and other myasthenic syndromes (24\%), epilepsy and genetic epilepsy (20\%) and functional neurological disorders (16\%). The high percentage of patients with myasthenia gravis in the study can be attributed to the myasthenia gravis community heavily promoting the survey.

Of the patients surveyed, $131(65 \%)$ had participated in remote consultations. Of the $70(35 \%)$ that had yet to 

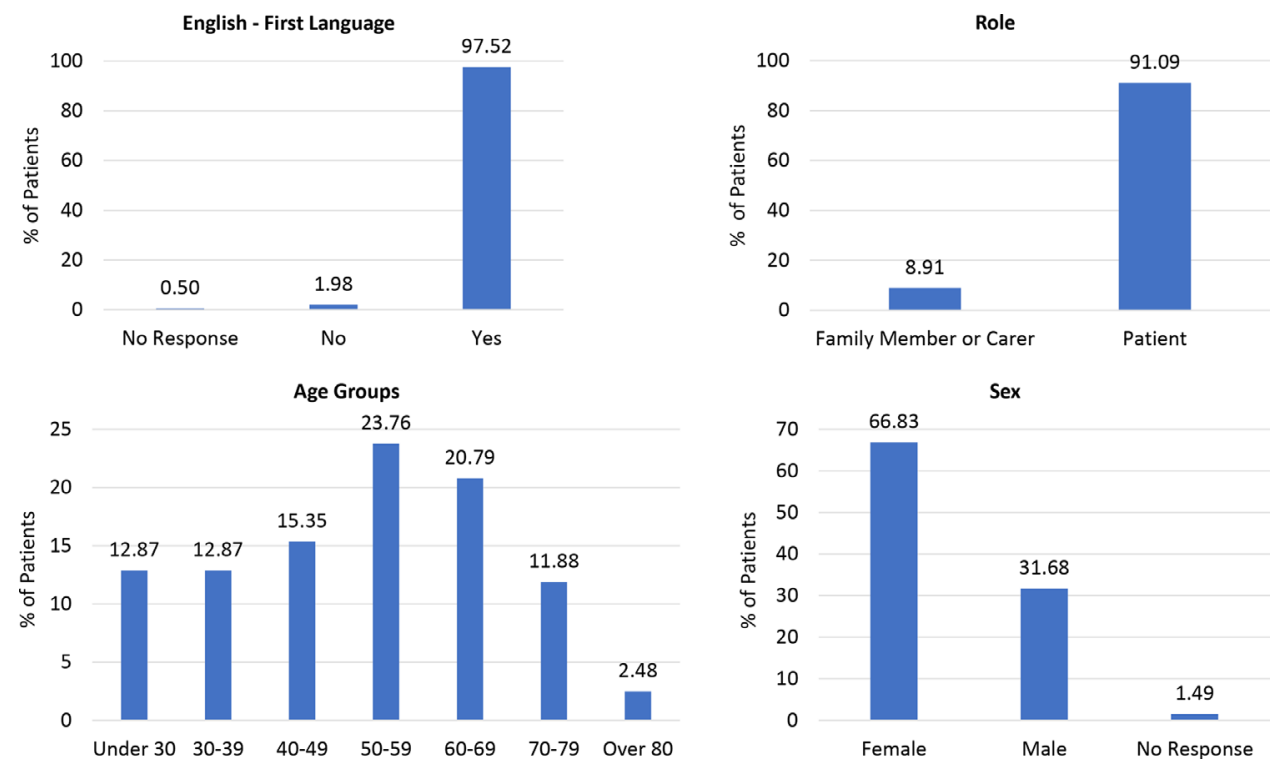

Figure 1 Patient demographics.

experience a remote consultation, $77 \%$ expressed interest in remote consulting as part of their future follow-up (see online supplemental figures 1 and 2 for further information on this cohort).

In the cohort who had participated in remote consultation(s), $103(79 \%)$ had received phone appointments only, $11(8 \%)$ had received video appointments only and $17(13 \%)$ had received both. Patient preferences regarding consultation types (phone; video; phone and video) are illustrated in figure 3. Although $43 \%$ of patients reported a preference for video consultations, only $20(10 \%)$ patients had actually undergone a video consultation themselves. Moreover, only $3(11 \%)$ of the patients who experienced a video consultation reported a preference for phone consultations. Multivariable modelling revealed that consultation preference differed according to the underlying neurological condition (figure 4). For example, patients with FND and Parkinson's disease had a higher preference for video consultation compared with those with epilepsy and genetic epilepsy $(9.53,95 \%$ CI 1.29 to 70.33 and 10.73 , 95\% CI 1.04 to 110.54 respectively).

The majority of patients perceived remote consultations as safe, useful, effective and convenient, with $88 \%$ of

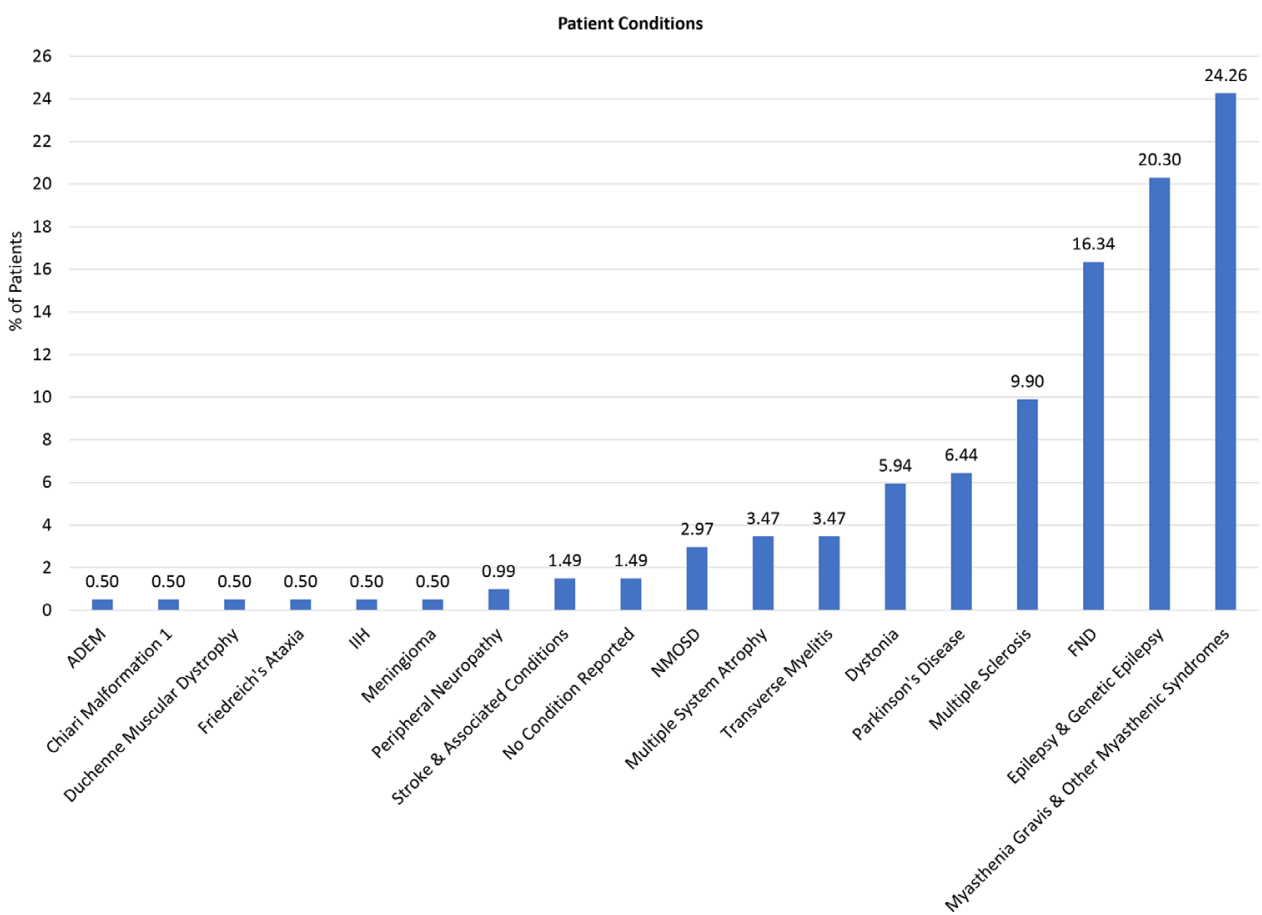

Figure 2 Patient conditions. ADEM, acute disseminated encephalomyelitis; FND, functional neurological disorder; IIH, idiopathic intracranial hypertension; NMOSD, neuromyelitis optica spectrum disorder. 

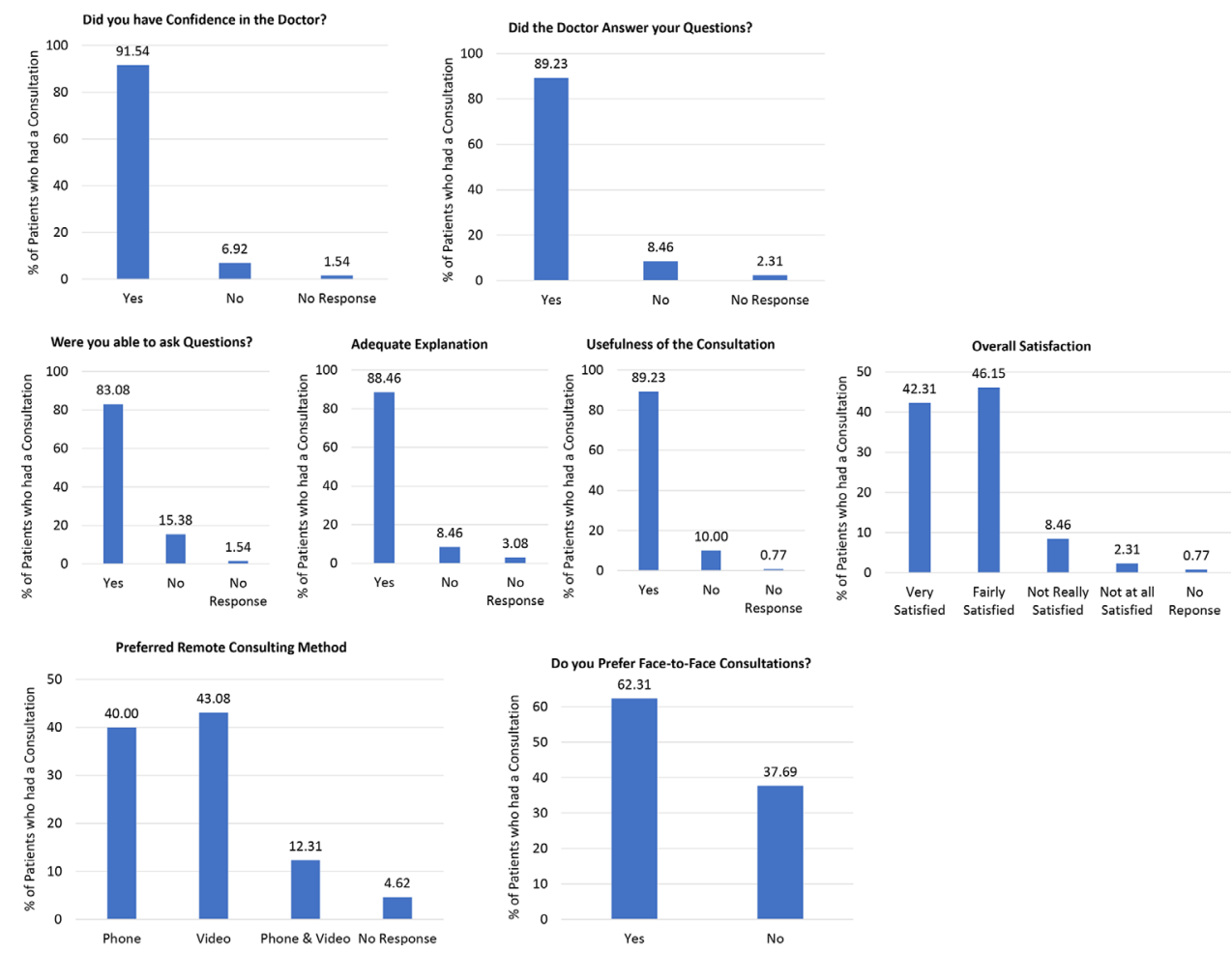

Figure 3 Patient views on remote consulting.

patients being overall satisfied with their remote consultation experience (figure 3, online supplemental figure $3)$. Interestingly, despite the survey taking place during a national infection outbreak, only $50.5 \%$ reported 'reduced risk of infection' as one of the positive aspects of remote consulting (online supplemental figure 3). This suggests that a lot of the positive perceptions of remote consultations for patients would still be applicable in a 'business-as-usual' setting after the COVID-19 outbreak is under control.

Multivariable logistic regression modelling demonstrated that age, sex and the underlying neurological condition were important determinants of patients' satisfaction ratings (figure 4). For instance, patients with Parkinson's disease had significantly lower satisfaction compared with those with epilepsy and genetic epilepsy $(0.57,95 \%$ CI 0.33 to 0.96$)$. In addition, patients aged $>80$ years were associated with lower odds of satisfaction compared with other age groups. The lower satisfaction in the $>80$ age group may be attributed to the lack of technological proficiency, outlined in the introduction, which can sometimes be seen among some elderly patients. Male patients were more likely to report satisfaction with remote consultations compared with female patients $(1.38,95 \% \mathrm{CI} 1.06$ to 1.79$)$. Although traditional face-to-face consultations were the favoured way of interaction for $62 \%$ of patients, a significant proportion (60\%) preferred that at least some of their future consultations be performed remotely.

\section{Clinicians}

In total, 62 neurologists from a reasonably broad representation of the different centres across Scotland participated in the national survey, the majority (79\%) being consultants. All clinicians undertook phone appointments and 68\% used 'Attend Anywhere' / Near Me', a secure NHS video call platform. The majority of clinicians preferred phone over video (phone $63 \%$ vs video $33 \%, \mathrm{p}=0.003$ ), indicating that phone consultations were as effective and informative as video appointments, without the added technical challenges (for both patients and neurologists) of the latter. Phone appointments were largely the preferred consultation type as they were easier to organise, more widely available and reliable, and less technically complex. Although favoured by fewer clinicians overall, the stated benefits of video consultations included the enhanced ability for rapport-building through non-verbal communication and improved performance of clinical examination, even if limited.

The positive and negative aspects associated with remote consulting from the clinician perspective and the methods implemented to overcome the barriers are outlined in tables $1-3$, respectively. Ninety-five per cent of physicians stated that remote consulting is suitable and convenient for selected groups of patients including patients with mobility issues, those disengaged from healthcare with poor appointment attendance, patients with established and stable neurological conditions that do not require frequent clinical examination (eg, epilepsy, headache) and history-driven neurological conditions (eg, first seizure patients). Additionally, six physicians explicitly expressed that for new patients, face-to-face review is the gold standard and remote consulting would compromise diagnosis and management. Notably, most clinicians stated a preference for remote consultations 

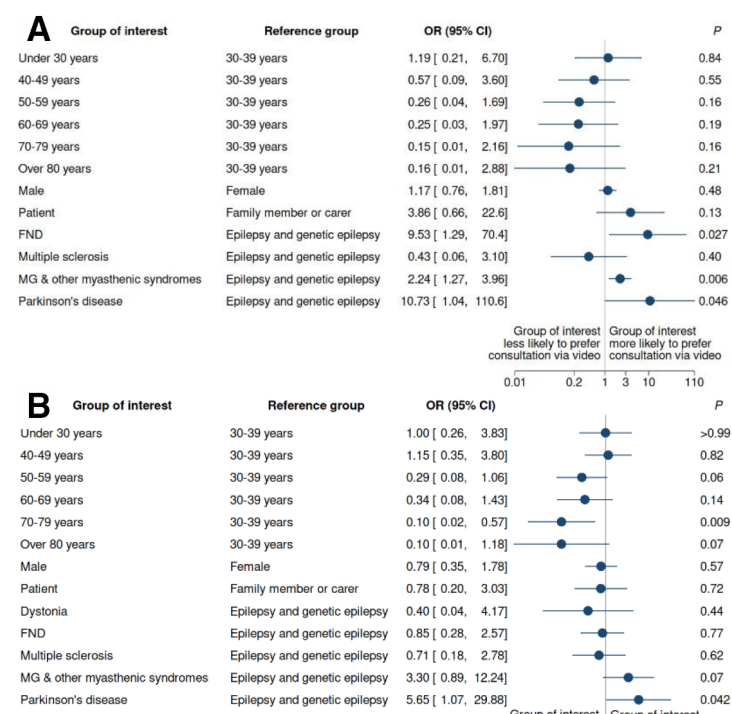

$0.29[0.08, \quad 1.06]$

$0.34[0.08, \quad 1.43]$

$0.10[0.02,0.57]$

$10[001,1181 \longrightarrow$

$0.79[0.35,1.78]$

$0.78[0.20,3.03]$

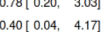

$0.85[0.28,2.57]$

$0.85\left[\begin{array}{ll}0.28, & 2.57\end{array}\right]$

3301089,1224

$565[107,2989$

Parkinson's disease

.65 [1.07, 29.88]
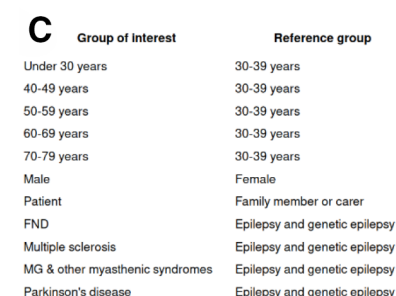

OR $195 \%$ C

OR $(95 \% \mathrm{Cl})$
$1.15[0.06,22.07]$

$0.95[0.05,17.38]$

$0.39[0.02, \quad 7.68]$

1.62[0.11, 24.25]

2.13[0.11, 41.47]

$0.67[0.12,3.70]$

$0.20[0.02,2.23]$

$2.24[0.20,25.37]$

$2.12[0.11,41.48$ ]

$2.13[0.12,37.36]$
$2.90[0.11,74.83]$

Parkinson's dise
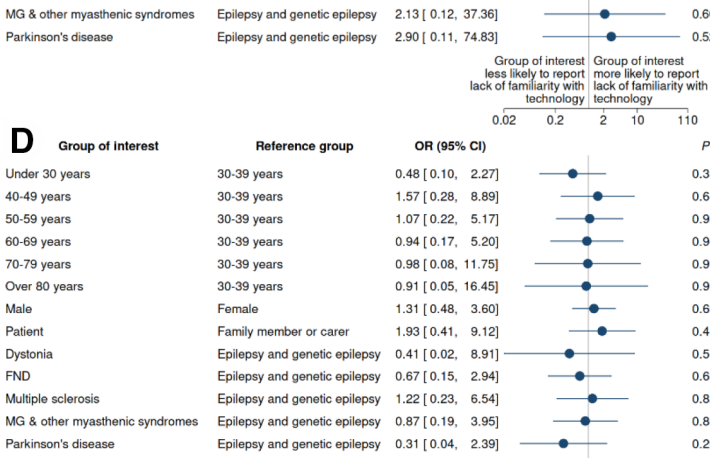

OR $(95 \%$

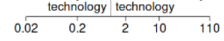

$0.48\left[\begin{array}{ll}0.10, & 2.27\end{array}\right]$

$1.57[0.28,8.89]$

$\begin{array}{lll}1.07[0.22, & 5.17]\end{array}$

$0.94[0.17, \quad 5.20]$

$0.98[0.08,11.75]$

$0.91[0.05,16.45]$
$1.31[0.48,3.60]$

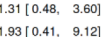

$1.93[0.41,9.12]$

$0.41[0.02,8.91]$

$0.67[0.15,2.94]$

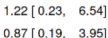

$0.87[0.19,3.95]$
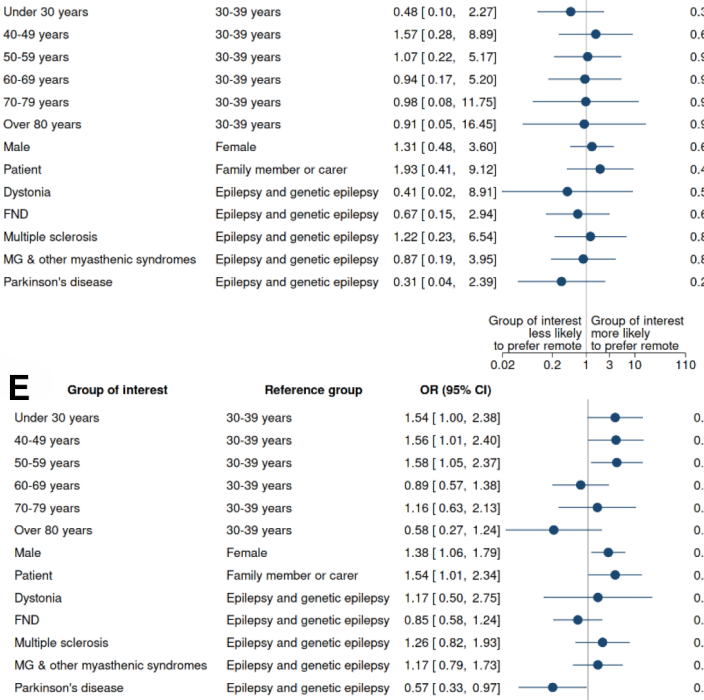

OR $(95 \%$ C

$1.54[1.00,2.38]$

$1.54[1.00,2.38]$
$1.56[1.01,2.40]$

$.56[1.01,2.40]$

$.58[1.05,2.37]$

$0.80 .57,1.38]$

$1.16[0.63,2.13]$
$0.58[0.27,1.24]$

$0.58[0.27,1.24]$
$1.38[1.06,1.79]$

$1.38[1.06,1.79]$
$1.54[1.01,2.34]$

1. $.54[1.01,2.34]$

$1.17[0.50,2.75]$
$0.85[0.58,1.24]$ $1.17[0.79,1.73]$ Epilepsy and genetic epileps

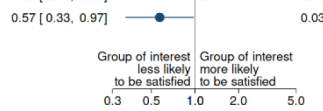

(a) Outcome: Consultation preference (video). OR denotes odds ratio. $95 \% \mathrm{Cl}$ denotes $95 \%$ confidence interval. MG denotes myasthenia gravis and FND denotes Functional Neurological Disorder. Results are based on a multivariable logistic regression model. An $O R>1$ indicates that the group of interest is more likely to prefer consultation via video - adjusting for all other variables in the model. Solid circles represent the OR. Horizontal lines depict $95 \%$ Cls around the point estimates. When the horizontal lines do not cross the vertical grey line (null effect, $O R=1.0$ ) results are considered statistically significant at alpha $=5 \%$.

(b) Outcome: Concerns about effectiveness of remote consultation. OR denotes odds ratio. $95 \% \mathrm{Cl}$ denotes $95 \%$ confidence interval. MG denotes myasthenia gravis and FND denotes Functional Neurological Disorder. Results are based on a multivariable logistic regression model. An OR > 1 indicates that the group of interest is more likely to report concerns about effectiveness of remote consultation adjusting for all other variables in the model. Solid circles represent the OR. Horizontal lines depict $95 \% \mathrm{Cls}$ around the point estimates. When the horizontal lines do not cross the vertical grey line (null effect, $O R=1.0$ ) results are considered statistically significant at alpha $=5 \%$.

(c) Outcome: Lack of familiarity with technology. OR denotes odds ratio. $95 \% \mathrm{Cl}$ denotes $95 \%$ confidence interval. MG denotes myasthenia gravis and FND denotes Functional Neurological Disorder. Results are based on a multivariable logistic regression model. An $O R>1$ indicates that the group of interest is more likely to report lack of familiarity with technology - adjusting for all other variables in the model. Solid circles represent the OR. Horizontal lines depict $95 \% \mathrm{Cls}$ around the point estimates. When the horizontal lines do not cross the vertical grey line (null effect, $O R=1.0$ ) results are considered statistically significant at alpha $=5 \%$.

(d) Outcome: Prefer remote. OR denotes odds ratio. $95 \% \mathrm{Cl}$ denotes $95 \%$ confidence interval. MG denotes myasthenia gravis and FND denotes Functional Neurological Disorder. Results are based on a multivariable logistic regression model. An $O R>1$ indicates that the group of interest is more likely to prefer remote consultation adjusting for all other variables in the model. Solid circles represent the OR. Horizontal lines depict $95 \% \mathrm{Cls}$ around the point estimates. When the horizontal lines do not cross the vertical grey line (null effect, $O R=1.0$ ) results are considered statistically significant at alpha $=5 \%$.

(e) Satisfaction. OR denotes odds ratio. $95 \% \mathrm{Cl}$ denotes $95 \%$ confidence interval. MG denotes myasthenia gravis and FND denotes Functional Neurological Disorder. Results are based on a multivariable logistic regression model. An $O R>1$ indicates that the group of interest is more likely to report satisfaction with remote consultation - adjusting for all other variables in the model. Solid circles represent the OR. Horizontal lines depict $95 \% \mathrm{Cls}$ around the point estimates. When the horizontal lines do not cross the vertical grey line (null effect, $O R=1.0$ ) results are considered statistically significant at alpha $=5 \%$.

Figure 4 Multivariable logistic regression models. (A) outcome: consultation preference (video); (B) outcome: concerns about effectiveness of remote consultation; (C) outcome: lack of familiarity with technology; (D) outcome: prefer remote; (E) satisfaction.

becoming incorporated into their future routine work (91\% vs $9 \%, \mathrm{p}<0.001)$.

Although most clinicians considered their jobs satisfying since the introduction of remote consulting ('satisfied' $63 \%$ vs 'not satisfied' $37 \%, \mathrm{p}=0.009$ ), this new model did not shorten consultation times compared with standard appointments ('did not shorten consultation' $53 \%$ vs 'did shorten consultation' $37 \%, \mathrm{p}=0.132$ ) and did not allow the completion of more evaluations ('did not complete more evaluations' $81 \%$ vs 'completed more evaluations' $19 \%, \mathrm{p}<0.001)$. Most neurologists were not confident about the quality and interpretation of their neurological assessments during a remote consultation ('not confident' $81 \%$ vs 'confident' $19 \%, \mathrm{p}<0.001)$. Overall clinician 
Table 1 Positive aspects of remote consulting for patients

Percent of clinician respondents

\begin{tabular}{ll} 
Positive aspect & respondents \\
\hline $\begin{array}{l}\text { Convenience/reduced travel time } \\
\text { for patients }\end{array}$ & $46 \%$ \\
$\begin{array}{l}\text { Comfortable environment for } \\
\text { patients }\end{array}$ & $10 \%$ \\
$\begin{array}{l}\text { Easier to involve family members/ } \\
\text { carers/witness accounts/translators }\end{array}$ & $4 \%$ \\
\hline $\begin{array}{l}\text { More efficient } \\
\text { More flexible }\end{array}$ & $10 \%$ \\
\hline Decreased risk of infection & $6 \%$ \\
\hline
\end{tabular}

productivity and flexibility in organising their working hours did not improve ( $(82 \%$ vs $18 \%, \mathrm{p}<0.001)(53 \%$ vs $47 \%, \mathrm{p}=0.708)$, respectively).

\section{Teleneurology and the future of neurology training}

We also explored clinicians' views on the impact of teleneurology on training. Table 4 summarises the advantages and disadvantages of introducing remote consulting into the neurology training curriculum. The prevailing opinion was that considerable ongoing effort is required to adapt and align structures, processes and training to successfully embed this new model of care.

Most physicians felt that training interventions for remote consultation skills are required to improve clinician consultation skills ('yes' $63 \%$ vs 'no' $37 \%, \mathrm{p}=0.009$ ). Clinical exposure to both remote and face-to-face consultations should be a prerequisite for satisfactory completion of training, with nine physicians expressing that such consultations should be carried out under the guidance of the supervising consultant. Specific training interventions such as dedicated training days (formal training on communication skills and clinical assessments adapted to remote consulting) and the development of standardised guidelines were suggested by 14 respondents as two ways

Table 2 Negative aspects/barriers of remote consulting for patients

\begin{tabular}{ll}
\hline Negative aspect & $\begin{array}{l}\text { Number of } \\
\text { clinician } \\
\text { respondents }\end{array}$ \\
\hline $\begin{array}{l}\text { Developing rapport with patients } \\
\text { Technological challenges }\end{array}$ & $72 \%$ \\
$\begin{array}{l}\text { Challenges with history and neurological } \\
\text { examination }\end{array}$ & $91 \%$ \\
\hline $\begin{array}{l}\text { Loss of control over consultations } \\
\text { Interpreting patient's expectations/ } \\
\text { satisfaction }\end{array}$ & $5 \%$ \\
$\begin{array}{l}\text { Duplication of work (incorrect patient } \\
\text { contact details/ unanswered/missed calls, } \\
\text { arranging face-to-face consultation) }\end{array}$ & $28 \%$ \\
\hline
\end{tabular}

in which to help trainees and consultants conduct remote consultations efficiently.

\section{DISCUSSION}

Although not a replacement for face-to-face consultations, this survey supports the notion that remote consulting can be an acceptable adjunct for doctors and patients. To our knowledge, this is the first in-depth analysis of the use of remote consulting in neurological outpatient clinics in Scotland during the COVID-19 pandemic and provides timely and important evidence on both patient and clinician experiences. NHS Scotland is currently promoting the 'Attend Anywhere'/'Near Me' video consulting service, citing benefits including cost-effectiveness, reduced travel and increased accessibility. ${ }^{18}$ However, more research is required to identify the overall safety and applicability of this approach, and which groups of patients are most suited to remote consulting.

There were stark discrepancies in the preferred type and perceptions of remote consulting between patients and physicians. Although patients were enthusiastic about video consultations, the majority of patients received phone appointments as this type of remote consultation was the favoured method for clinicians. This preference of clinicians may be related to some internet connections being insufficient for "Attend Anywhere" consultations, which consequently often results in them switching to phone appointments. ${ }^{19}$ The contrary patient preference may be related to video consultations allowing greater familiarity and engagement with the physician through body language and non-verbal communication, such as eye contact, supporting the patient's confidence and trust in the physician. ${ }^{20}$

Additionally, the satisfaction and subjective success of remote consulting differed between the two groups. Most clinicians (72\%) described obstacles around establishing patient rapport and therapeutic relationships during remote consultations, while the advantages, such as the effect on overall workload, were less clear. This is in contrast to prior studies that reported numerous benefits for physicians, including time saving and fewer missed appointments and cancellations. ${ }^{32}$ Nevertheless, similar findings regarding challenges with technology and subsequent impairment in the consultation quality have been reported in other studies. ${ }^{31922}$

The majority of patients surveyed expressed satisfactory communication with their clinicians-citing adequate explanations $(88 \%)$ and competent responses to their questions and concerns (89\%) - which may have contributed towards the high overall patient satisfaction. This relates to the findings of Elliott et al, ${ }^{23}$ who concluded that the factors most associated with high patient satisfaction were the physician's capability to build rapport, share information and provide guidance. Despite the differing views between clinicians and patients on remote consulting, both groups agreed that routine remote consultations should be implemented in the future. 
Table 3 Methods implemented to overcome barriers during remote consulting

\begin{tabular}{llc} 
Barrier & Method used to overcome barrier & $\begin{array}{l}\text { Number of clinician respondents } \\
\text { using each method }\end{array}$ \\
\hline $\begin{array}{l}\text { Patients with communication } \\
\text { (deafness/dysarthria) or cognitive } \\
\text { difficulties }\end{array}$ & $\begin{array}{l}\text { Involvement of caregiver/next of kin } \\
\text { Arrangement of face-to-face consultation }\end{array}$ & $55(95 \%)$ \\
Patients who speak only limited & Involvement of caregiver/next of kin & $42(70 \%)$ \\
English & Involvement of interpreter & $35(58 \%)$ \\
& $(1)$ Easy to access & $24(69 \%)$ \\
& $(2)$ Not easy to access & $11(31 \%)$ \\
& Arrangement of face-to-face consultation & $3(5 \%)$ \\
Patients who are difficult to reach & Speaking to patient's next of kin/carers/nursing & $21(51 \%)$ \\
& staff/nominated others & $1(2 \%)$ \\
& Ringing repeatedly & $15(37 \%)$ \\
& Using telephone appointments rather than video & $1(2 \%)$ \\
& Involvement of patient's GP & $5(12 \%)$ \\
\hline
\end{tabular}

Anecdotally, we have become aware that some patients do not regard remote consulting as a 'proper' assessment, more of a pandemic related holding mechanism; we found little evidence in our survey however to support this impression.

\section{Limitations of the survey}

There are several limitations of our study, and we acknowledge that our study only portrays opinions from a snapshot in time. We plan to conduct a repeat survey and possibly an audit before drawing any firm conclusions. First, 98\% of the patients surveyed were native English speakers. As such, involvement from interpreters and third-party persons was generally not necessary, which could have contributed to the overwhelmingly positive patient experiences of remote consulting in this survey. Language barriers and miscommunication inevitably impacts the effectiveness and equity of healthcare and such barriers / discrepancies may be amplified in remote consulting.
For remote consulting to have a future in routine healthcare, it needs to be able to serve the whole community, including those who might not be fluent in the society's primary language. Considering that individuals with neurological conditions frequently have communication disorders (ie, speech and hearing disabilities), research into the infrastructure requirements to support this patient cohort is necessary for effective remote consulting to be implemented more extensively. ${ }^{24}$ This may include remote interpretation services being available and easily accessible to physicians when needed. ${ }^{25}$

Additionally, certain patient groups were overrepresented in the data (ie, individuals with myasthenia gravis and other myasthenic syndromes account for $24 \%$ of respondents and those with epilepsy/genetic epilepsy comprise 20\%); as such, the neurological conditions of the patients included in this study are not accurately representative of the variety of neurological patients

Table 4 Advantages and disadvantages of introducing remote consulting in neurology training

\begin{tabular}{|c|c|}
\hline Advantages reported by clinicians & Disadvantages reported by clinicians \\
\hline Gaining familiarity with technology & Reduced clinical exposure \\
\hline Development and improvement of remote consultation skills & $\begin{array}{l}\text { Lack of development of rapport with patients and ability to } \\
\text { interpret body language }\end{array}$ \\
\hline Better appreciation of challenges around remote consulting & Lack of communication skills development \\
\hline Preparation for their future consultant roles & Reduced practice of examination skills \\
\hline Continuous training during COVID-19 restrictions & Increased risk of misdiagnosis \\
\hline \multirow{4}{*}{$\begin{array}{l}\text { Assessment of patients that would otherwise only be seen by } \\
\text { GP/other specialties }\end{array}$} & Overinvestigating patients \\
\hline & Duplication of work/unnecessary follow-up appointments \\
\hline & Difficulty to organise supervision/inadequate supervision \\
\hline & Unsuitability for new patients \\
\hline
\end{tabular}


encountered in the community. The high number of patients with myasthenia gravis participating in this study may reflect the enthusiasm of this patient cohort and implicitly suggest that patients with myasthenia gravis may be relatively more amenable to remote reviews. Such findings could be attributed to greater promotional activity by the corresponding charitable organisations, which led to increased awareness and completion of the online survey in these cohorts. We also need to acknowledge that the patients who responded to the online survey are likely the ones who are more technologically proficient and familiar with web-based applications and those with less severe forms of neurological conditions; these factors may have also influenced the outcomes.

Finally, the survey was conducted from October to November 2020 and therefore provides a snapshot of experiences during the pandemic. Factors such as improved telehealth care systems, acclimatisation to the new clinic workflow and navigation through the telehealth platforms by patients and clinicians-but also pandemic and digital fatigue on the other side-would inevitably affect the outcome profile when re-implementing the survey.

\section{Implications for practice and neurology training}

The prevailing consensus among neurologists was that effective and safe care can be delivered remotely, when appropriate. However, face-to-face review is of particular importance for new patients as remote consulting could compromise initial diagnosis and management. To successfully implement this new model of care on a broader scale, additional effort is needed to optimise structures and training within the neurology specialty. For example, further research, such as randomised controlled trials, are urgently required to investigate the role of different types of remote consulting in neurology; in terms of suitability of patients and conditions, use in acute and follow-up presentations and impact on resource utilisation. Furthermore, joint efforts from multiple stakeholders will be necessary for the development of platforms that permit a holistic approach by reducing disparities in healthcare access, such as communication barriers.

As remote consulting is a developing form of healthcare, most neurologists reported the necessity of incorporating education in virtual care to neurology training or continuing education curricula. Despite the growing clinical relevance and worldwide implementation of remote consulting, standard recommendations about the necessary components for an effective and comprehensive remote consulting curriculum are yet to be agreed/ published. ${ }^{26-28}$

Stovel $e t a l^{28}$ determined that successful remote consulting-training curricula should involve hands-on experiences and the use of multiple teaching modalities such as simulation, interactive case-based sessions and opportunities for reflection. This training content should also have a particular focus on developing existing clinical and communication skills for a setting that uses online technology. To ensure the delivery of safe and effective care through teleneurology, further research is needed to determine standardised guidelines for high-quality practice, along with an appropriate and thorough training curriculum to achieve this.

Acknowledgements We thank the Neurological Alliance of Scotland and the people with neurological conditions for their support in implementing this survey.

Contributors MS designed the study protocol and the web-based surveys, extracted and interpreted data, performed the statistical analysis, produced tables and composed the manuscript and is acting as guarantor. EL and JL extracted and interpreted data, composed the manuscript and produced tables and figures. RJD contributed to the data analysis and manuscript revision.

Funding The authors have not declared a specific grant for this research from any funding agency in the public, commercial or not-for-profit sectors.

Competing interests None declared.

Patient consent for publication Not applicable.

Provenance and peer review Not commissioned; externally peer reviewed.

Data availability statement All data relevant to the study are included in the article or uploaded as supplementary information.

Supplemental material This content has been supplied by the author(s). It has not been vetted by BMJ Publishing Group Limited (BMJ) and may not have been peer-reviewed. Any opinions or recommendations discussed are solely those of the author(s) and are not endorsed by BMJ. BMJ disclaims all liability and responsibility arising from any reliance placed on the content. Where the content includes any translated material, BMJ does not warrant the accuracy and reliability of the translations (including but not limited to local regulations, clinical guidelines, terminology, drug names and drug dosages), and is not responsible for any error and/or omissions arising from translation and adaptation or otherwise.

Open access This is an open access article distributed in accordance with the Creative Commons Attribution Non Commercial (CC BY-NC 4.0) license, which permits others to distribute, remix, adapt, build upon this work non-commercially, and license their derivative works on different terms, provided the original work is properly cited, appropriate credit is given, any changes made indicated, and the use is non-commercial. See: http://creativecommons.org/licenses/by-nc/4.0/.

\section{REFERENCES}

1 Davis LE, Coleman J, Harnar J, et al. Teleneurology: successful delivery of chronic neurologic care to 354 patients living remotely in a rural state. Telemed J E Health 2014;20:473-7.

2 Hatcher-Martin JM, Adams JL, Anderson ER, et al. Telemedicine in neurology: telemedicine work group of the American Academy of Neurology update. Neurology 2020;94:30-8.

3 Patel UK, Malik P, DeMasi M, et al. Multidisciplinary approach and outcomes of tele-neurology: a review. Cureus 2019;11:4410.

4 Wechsler LR, Tsao JW, Levine SR, et al. Teleneurology applications: report of the telemedicine work group of the American Academy of Neurology. Neurology 2013;80:670-6.

5 Guzik AK, Switzer JA. Teleneurology is neurology. Neurology 2020;94:16-17.

6 Bladin CF, Cadilhac DA. Effect of telestroke on emergent stroke care and stroke outcomes. Stroke 2014;45:1876-80.

7 Campos C, Caudevilla E, Alesanco A, et al. Setting up a telemedicine service for remote real-time video-EEG consultation in La Rioja (Spain). Int J Med Inform 2012;81:404-14.

8 Dorsey ER, Deuel LM, Voss TS, et al. Increasing access to specialty care: A pilot, randomized controlled trial of telemedicine for Parkinson's disease. Mov. Disord. 2010;25:1652-9.

9 Dorsey ER, Venkataraman V, Grana MJ, et al. Randomized controlled clinical trial of "virtual house calls" for Parkinson disease. JAMA Neurol 2013;70:565-70.

10 Dorsey ER, Wagner JD, Bull MT, et al. Feasibility of Virtual Research Visits in Fox Trial Finder. J Parkinsons Dis 2015;5:505-15.

11 Turner AP, Sloan AP, Kivlahan DR, et al. Telephone counseling and home telehealth monitoring to improve medication adherence: results of a pilot trial among individuals with multiple sclerosis. Rehabil Psychol 2014;59:136-46.

12 Qiang JK, Marras C. Telemedicine in Parkinson's disease: a patient perspective at a tertiary care centre. Parkinsonism Relat Disord 2015;21:525-8. 
13 George BP, Scoglio NJ, Reminick JI, et al. Telemedicine in leading US neurology departments. Neurohospitalist 2012;2:123-8.

14 Schneider RB, Biglan KM. The promise of telemedicine for chronic neurological disorders: the example of Parkinson's disease. The Lancet Neurology 2017;16:541-51.

15 Ohannessian R, Duong TA, Odone A. Global telemedicine implementation and integration within health systems to fight the COVID-19 pandemic: a call to action. JMIR Public Health Surveill 2020;6:e18810.

16 Neurological Alliance of Scotland. About us [Internet], 2021. Available: https://www.scottishneurological.org.uk/about/ [Accessed 31 Mar 2021].

17 Scottish Government. Coronavirus (COVID-19) update: First Minister's statement - 4 January 2021 [Internet], 2021. Available: https://www.gov.scot/publications/coronavirus-covid-19-updatefirst-ministers-statement-monday-4-january-2021/ [Accessed 31 Mar 2021].

18 Wherton J, Greenhalgh T. Evaluation of the attend Anywhere/Near me video consulting service in Scotland, 2019-20. Scottish Government, 2020.

19 Hammersley V, Donaghy E, Parker R, et al. Comparing the content and quality of video, telephone, and face-to-face consultations: a non-randomised, quasi-experimental, exploratory study in UK primary care. Br J Gen Pract 2019;69:e595-604.

20 Horton T, Jones B. Three key quality considerations for remote consultations [Internet]. The Health Foundation, 2020. Available: https://www.health.org.uk/news-and-comment/blogs/three-key- quality-considerations-for-remote-consultations [Accessed $27 \mathrm{Mar}$ 2021].

21 Gomez T, Anaya YB, Shih KJ, et al. A qualitative study of primary care physicians' experiences with telemedicine during COVID-19. $J$ Am Board Fam Med 2021;34:S61-70.

22 Donaghy E, Atherton H, Hammersley V, et al. Acceptability, benefits, and challenges of video consulting: a qualitative study in primary care. British Journal of General Practice 2019;69:e586-94.

23 Elliott T, Tong I, Sheridan A, et al. Beyond convenience: patients' perceptions of physician Interactional skills and compassion via telemedicine. Mayo Clin Proc Innov Qual Outcomes 2020;4:305-14.

24 Annaswamy TM, Verduzco-Gutierrez M, Frieden L. Telemedicine barriers and challenges for persons with disabilities: COVID-19 and beyond. Disabil Health J 2020;13:100973.

25 Walsh B. Breaking the language barrier in telemedicine [Internet]. Axios, 2020. Available: https://www.axios.com/telehealthcoronavirus-language-barriers-7fd5be68-3551-464d-ada90c1441197bbb.html [Accessed 27 Mar 2021].

26 Basu A, Seaton P, Kirk R, et al. Review of the effectiveness of educational tools for teaching telehealth care. Christchurch: University of Canterbury, 2010.

27 Edirippulige S, Armfield NR. Education and training to support the use of clinical telehealth: a review of the literature. $J$ Telemed Telecare 2017;23:273-82.

28 Stovel RG, Gabarin N, Cavalcanti RB, et al. Curricular needs for training telemedicine physicians: a scoping review. Med Teach 2020;42:1234-42. 